\title{
THE USE OF MANAGEMENT TOOL AS FACILITATOR OF THE INDUSTRIAL MAINTENANCE PLAN
}

\author{
Jefferson de Souza Paiva1, Renato Brasil Sodré2, Anderson de Oliveira Castro ${ }^{3}$
}

${ }^{1}$ Acadêmico de Engenharia Mecânica, Uninorte Laureate

${ }^{2}$ Acadêmico de Engenharia Mecânica, Uninorte Laureate

${ }^{3}$ Mestre em Engenharia Elétrica, Uninorte Laureate

Email: firmezzaz@gmail.com, renatosodre28@gmail.com, anderson.castro@uninorte.com.br

Received: June $03^{\text {th }}, 2019$

Accepted: June $06^{\text {th }}, 2019$

Published: September $30^{\text {th }}$, 2019

Copyright @ 2016 by authors and Institute of Technology Galileo of Amazon (ITEGAM). This work is licensed under the Creative Commons Attribution International License (CC BY 4.0). https://creativecommons.org/lice nses/by/4.0/

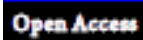

\begin{abstract}
The present article was elaborated through bibliographical research done in books, websites and scientific articles. The objective was to present the importance of the management tool as a facilitator of the preventive maintenance plan in a manufacturing environment. Management tools such as Lean Manufacturing, Gantt Chart and Maintenance Performance Indicators were analyzed, which contributed to the implementation of the preventive plan. The tools studied in this work, aid in planning and integrated reliability within the plant, and enables a preventive maintenance intervention in the production processes, contributing with on-time delivery of products with good quality and production volume, availability of machines and cost minimization.
\end{abstract}

Keywords: Preventive Maintenance, Maintenance Plan, Performance Indicator

\section{O USO DE FERRAMENTA DE GESTÃO COMO FACILITADOR DO PLANO DE MANUTENÇÃO INDUSTRIAL}

\section{RESUMO}

O presente artigo foi elaborado por meio de pesquisas bibliográficas realizadas em livros, sites e artigos científicos. O objetivo foi apresentar a importância da ferramenta de gestão como facilitadora do plano de manutenção preventiva num ambiente fabril. Foram analisadas as ferramentas de gestão, como Lean Manufacturing, Gráfico de Gantt e Indicadores de Desempenho da manutenção, os quais contribuíram na aplicação do plano preventivo. As ferramentas estudadas neste trabalho, auxiliam no planejamento e na confiabilidade integrada dentro da unidade fabril, e possibilita uma intervenção da manutenção preventiva nos processos de produção, contribuindo com entrega de produtos dentro do prazo, com boa qualidade e volume de produção, disponibilidade de máquinas e minimização de custos.

Palavras-chave: Manutenção Preventiva, Plano de Manutenção, Indicador de Desempenho

\section{INTRODUÇÃO}

Em uma organização é importante manter os equipamentos em boas condições para se obter maior lucratividade. A manutenção preventiva proporciona ao equipamento um melhor desempenho. Ocorre periodicamente, e os fatores que determinam sua frequência são a criticidade dos serviços e a disponibilidade da empresa.

A manutenção e um conjunto de ações de gestão, técnicas e econômicas, com o objetivo de otimizar o ciclo de vida útil dos bens de uma empresa [1]. É uma das áreas que mais influência na produtividade e qualidade, a manutenção atua nos equipamentos e 
máquinas produtivas e assim sua eficiência é um influenciador direto tanto na qualidade do produto quanto na quantidade produzida.

Dessa forma, observa-se que nos últimos anos a função manutenção se expandido e vários métodos de gestão surgiram, com objetivo de proporcionar ganhos em áreas variadas como maior segurança do operador, do equipamento, maior qualidade, maior produtividade, reduzir custos e reduzir impactos ambientais.

Com isso, o avanço da área de manutenção veio proporcionar a evolução de áreas de desenvolvimento tecnológicos e científicos, com o objetivo de atingir maior segurança em seus processos, garantindo um fluxo de atividades sem agressão ao meio ambiente, atendendo a qualidade exigida pelo mercado de produtos e serviços, a custos baixos e otimizados.

Para soluções de problemas de manutenção, podem ser utilizadas as ferramentas Lean Manufacturing, Gráfico de Gantt e Indicadores de desempenho da manutenção, beneficiando a confiabilidade dos equipamentos e o conhecimento técnico dos funcionários.

Uma observação muito importante em relação à manutenção preventiva é que ela se realize em dias fixos, objetivando uma programação mais criteriosa, com pessoas especializadas e consequentemente diminuindo os erros, alinhando os trabalhos que já se encontram na preventiva ou mesmo os que se tornaram criteriosos com o decorrer do tempo.

Este trabalho tem por objetivo explorar a importância da manutenção preventiva e aplicação das ferramentas de gestão na indústria, mostrando como podem ser vantajosas a aplicação das ferramentas em ganho de produtividade, qualidade e preservação dos equipamentos.

\section{HISTÓRICO E EVOLUÇÃO DA MANUTENÇÃO}

A manutenção, de acordo com [2], é uma palavra que deriva do latim manus tenere, que significa manter o que se tem, e está presente na história humana, desde o momento em que começamos a manusear instrumentos de produção. Surgiu efetivamente como função do organismo produtivo no século XVI, com a aparição dos primeiros teares mecânicos, uma época marcada por um período onde o fabricante do maquinário treinava os "novos operários" a operar e manter o equipamento, ocupando estes o papel de operadores e mantenedores, pois não havia uma equipe específica de manutenção.

Segundo [2], por volta de 1900, surgiram as primeiras técnicas de planejamento de serviços. No entanto, foi durante a Segunda Guerra Mundial que a manutenção se firmou como necessidade absoluta, quando houve então um fantástico desenvolvimento de técnicas da organização, planejamento e controle para tomada de decisão.

De acordo com [3], após a implementação da linha de montagem, por Henry Ford em 1913, houve a necessidade da criação de equipes para efetuar reparos assim que acontecesse a quebra. Foi a partir daí que surgiu o conceito de Manutenção Corretiva. Logo depois da Segunda Guerra Mundial, com o avanço das técnicas de produção, as intervenções corretivas já não eram mais suficientes e, por esse motivo, surgiu o conceito de Manutenção Preventiva para auxiliar na correção de falhas e evitar que elas ocorressem.

A aparição efetiva do termo "Manutenção", indicando a função de manter em bom funcionamento todo e qualquer equipamento, ferramenta ou dispositivo, ocorre na década de 1950 nos EUA, e neste mesmo período na Europa tal termo ocupa aos poucos os espaços nos meios produtivos, em detrimento da palavra "conservação" [2].

\section{Tipos de Manutenção}

[2] cita e classifica os tipos de manutenção:

Manutenção Corretiva;

Manutenção Preventiva;

Manutenção Preditiva;

Manutenção Autônoma.

Segundo [4], entende-se com o termo "manutenção" todas as medidas necessárias para manter/reestabelecer as condições específicas dos meios técnicos de um sistema, como também determinar e avaliar as condições existentes desse meio (...)”.

\section{Manutenção Corretiva}

Segundo [5], "Manutenção Corretiva é a representação do princípio, em que os mecânicos simplesmente consertavam o que estava quebrado, não se preocupando com as causas ou efeitos que ocasionaram o defeito".

Ela se aplica em emergência, expondo o funcionário a riscos devido à falta de segurança, pois não se sabe a causa raiz, e muitas vezes tendo pouco tempo para diagnosticar o problema, o que pode afetar a produtividade da indústria e gerar custos não planejados, até mesmo no caso em que não haja ferramentas em mãos [6].

\section{Manutenção Preventiva}

[7] afirmam que a manutenção preventiva é o estágio inicial da manutenção programada e deve obedecer a um padrão, estabelecendo paradas periódicas com o objetivo de executar os reparos devidamente programados e assim poder prolongar o tempo de vida útil de uma máquina.

Manutenção Preventiva é a "manutenção efetuada em intervalos predeterminados, ou de acordo com critérios prescritos, destinada a reduzir a probabilidade de falha ou a degradação do funcionamento de um item", ou seja, por meio de um plano de manutenção bem elaborado, a empresa consegue antecipar as falhas que possam ocorrer nos equipamentos [8].

\section{Manutenção Preditiva}

A Manutenção Preditiva é o tipo de manutenção em que é possível determinar ciclos, ou seja, determinar de quanto em quanto tempo se deve abrir uma máquina. São registrados, analisados e criados históricos de todos os dados referentes à máquina, conseguindo assim, prever quando acontecerão determinadas falhas [5].

\section{Manutenção Autônoma}

A Manutenção Autônoma é adotada pelos próprios operadores que passam a executar serviços de manutenção no maquinário que operam. Serviços estes que vão desde as instruções de limpeza e lubrificação, até serviços mais complexos de análise e melhoria dos instrumentos de produção [2].

Segundo [9], a manutenção autônoma possui sete etapas:

Etapa 1 - Limpeza Inicial: Limpeza e inspeção das máquinas e equipamentos;

Etapa 2 - Eliminação das Fontes de Sujeira e Locais de Difícil Acesso: Foco nas fontes de sujeira que possam vir a contaminar o operador ou até mesmo o ambiente de trabalho;

Etapa 3 - Padrões de Limpeza e Lubrificação: Busca pelo padrão ideal de inspeção e lubrificação;

Etapa 4 - Inspeção Geral: Treinamento em manutenção básica das máquinas e equipamentos;

Etapa 5 - Inspeção Autônoma: Criação de procedimentos e listas de verificação; 
Etapa 6 - Organização e Ordem: Organização dos locais de trabalho e uso correto dos recursos necessários;

Etapa 7 - Consolidação da Manutenção Autônoma: Consolidação das atividades por meio de uma programação anual de verificação das etapas.

"Sendo assim, a manutenção autônoma significa mudar a mentalidade para: "Deste equipamento, cuido eu", deixando de usar o antigo, que era: "Eu fabrico, você conserta"” [10].

\section{Gestão da Manutenção}

O modelo "TPM - Total Productive Maintenance" (Manutenção Produtiva Total) há três décadas tem se desenvolvido nas industrias em nível mundial, este modelo focado em custo/eficácia da manutenção, conduz a indústria a ganhos em disponibilidade de equipamentos e máquinas, reduzindo as manutenções corretivas emergenciais e aumentando a segurança do equipamento e da capacidade produtiva [1].

No Brasil há industrias que seguem o modelo de gestão tradicional e departamentalizada, mas com a competitividade do mercado que vem se elevando, onde as empresas maiores anexam as menores, em pouco tempo estas indústrias brasileiras conhecerão o TPM e sua filosofia, também outras como Gráfico de Gantt e indicadores de desempenho, que auxiliam na programação e planejamento de manutenções, principalmente em sistemas complexos onde o custo manutenção é elevado e o serviço toma dias de equipamento parado sem produzir [1]

\section{Lean Manufacturing e Indicadores de Desempenho da Manutenção \\ As políticas de manutenções têm passado por mudanças,} tempos atrás a manutenção era vista como necessária, hoje é um potencial aliado a segurança do processo produtivo, manutenção corretiva de reação imediata é evitada, e manutenções programadas proativas são preferenciais. Estabilizar o processo produtivo e confiabilidade na produção é o que se busca com estas mudanças nas políticas de manutenção [11].

\section{Lean Manufacturing}

O grande objetivo da filosofia Lean Manufacturing (Produção Magra) consiste na eliminação das sete grandes perdas associadas aos processos produtivos [11]:

Produção em excesso, ou seja, que ultrapassa o volume de encomendas;

Tempos de espera, entre as várias etapas de produção;

Transporte desnecessário de produtos fabricados e de matérias-primas e ferramentas, entre os vários locais de fabricação;

Processamento defeituoso, contribuindo para um reprocessamento e, eventualmente, para a quebra de confiança dos clientes;

Existência de stocks em excesso, no que respeita tanto aos produtos fabricados como aos materiais de manutenção, obrigando a perdas de tempo no armazenamento e no processamento administrativo e logístico, e à existência de armazéns sobre dimensionados face às necessidades;

Movimentações desnecessárias por parte dos recursos humanos;

Fabricação de produtos sem qualidade devida à fraca formação dos operadores dos equipamentos, e à ausência de especificações técnicas.

Esta metodologia é derivada do Sistema de Produção Toyota (TPS - Toyota Production System) e baseia-se em quatro grandes ferramentas de gestão [11]: Kaizen, Six Sigma, Kanban e Just in Time.

\section{Kaizen}

O kaizen visa o bem da empresa e também dos seus recursos humanos, que são motivados a contribuir com ideias e sugestões quem proporcionam a melhora do processo produtivo, os funcionários se sentem importantes e incentivados a colaborar diretamente no processo produtivo, apontando novas soluções e técnicas que melhorem os processos, reduzindo os desperdícios. Esta técnica permite igualmente definir os guias de operação para os operadores dos equipamentos e serve, ao mesmo tempo que os gestores usam a participação como método avaliativo [11].

Os conceitos do 5s são fáceis e simples de aplicar e baseiamse em boas práticas e a manter essas boas práticas. Porém, a implantação pode ser complicada, pois estas práticas nem sempre são aceitas de prontidão, outro fator e a cultura. O 5 s é sempre relacionado como a base do Lean Manufacturing.

A metodologia 5S surgiu de 5 palavras de origem japonesas, Seiei, Seiton, Seiso, Seiketsu e Shitsuke, que significam:

Seiri - organização e utilização - este primeiro S está focado em organizar o posto de trabalho com critério de utilização, facilitando o acesso do que usa diariamente e os benefícios com o primeiro $\mathrm{S}$ que é posto de trabalho organizado e rapidez ao encontrar o que se precisa.

O conceito básico desta metodologia consiste na qualidade de vida e de condições de trabalho dos recursos humanos, o que resulta numa maior produtividade obtida com maior qualidade. $\mathrm{O}$ Programa 5S surgiu das iniciais de cinco palavras de origem japonesa, Seiri, Seiton, Seiso, Seiketsu e Shitsuke, que têm os seguintes significados [12]:

Seiri - organização e utilização - consiste em manter no local de trabalho apenas o material, ferramentas e equipamentos estritamente necessários, separando assim o útil do inútil. Apresenta os seguintes benefícios:

1. Otimização do local de trabalho;

2. Maior rapidez na procura do material necessário;

3. Descarte do desnecessário e/ou do obsoleto;

4. Conhecer com exatidão o material existente.

Seiton - ordenação e arrumação - o material, as ferramentas e os equipamentos devem estar sempre organizados, isto é, cada peça deverá ter o seu local específico de arrumação. Apresenta os seguintes benefícios:

1. Diminuição dos riscos de acidentes;

2. Diminuição de stocks e de movimentação de peças e equipamentos;

3. Conhecimento real do material disponível;

4. Melhorias no controlo visual;

5. Rapidez na procura de peças.

Seiso - limpeza - ter sempre o cuidado de manter o local e o material de trabalho limpos, e procurar eventuais causas que possam diminuir o rendimento de trabalho. Apresenta os seguintes benefícios:

1. Redução dos custos de manutenção;

2. Aumento da motivação dos operários;

3. Aumento da vida útil das ferramentas e dos equipamentos;

4. Ambiente de trabalho mais agradável.

Seiketsu - padronização e higiene - manter todos os sectores da empresa com as mesmas características, através da combinação de Seiri, Seiton e Seiso. Apresenta os seguintes benefícios:

1. Maior entendimento entre os colaboradores da empresa;

2. Aumento da integração entre diferentes áreas;

3. Aumento do controlo visual;

4. Aumento do bem-estar dos colaboradores;

5. Aumento da motivação; 


\section{Optimização do tempo laboral.}

Shitsuke - autodisciplina - representa a autodisciplina através da interiorização de bons hábitos e de bons costumes, tratando igualmente da actualização constante

de conhecimentos por parte de todos os intervenientes no processo produtivo. Apresenta os seguintes benefícios:

1. Aumento das inter-relações;

2. Expansão da criatividade;

3. Cumprimento das normas de procedimentos definidos;

4. Consciencialização de valores éticos e morais;

5. Melhorias no desenvolvimento profissional;

6. Ascensão na carreira profissional;

7. Redução dos acidentes de trabalho.

\section{Six Sigma (Seis Sigma)}

O Six Sigma é aplicável em processos, produtos e serviços, com o objetivo de reduzir falhas e custos de produção, baseandose num forte sentido de disciplina e na melhoria contínua. O Six Sigma pretende atingir a meta "zero defeitos" através da prevenção de defeitos com base em ferramentas estatísticas. Neste sentido, avaliando as falhas ou defeitos de determinado processo industrial, de uma forma sistemática, é possível discernir quais os procedimentos de prevenção a adoptar para se eliminar essas falhas. Assim, esta metodologia focaliza-se na eliminação de desperdícios e na redução de defeitos, assim como na redução da variabilidade dos processos, recorrendo ao desvio padrão [11].

\section{Kanban (cartão)}

O procedimento Kanban é utilizado para descrever um sistema de sinalização muito simples, que autoriza a produção em cada célula de trabalho, a partir das operações a realizar a jusante. Os cartões kanban permitem estabelecer um controlo direto entre células de fabrico, limitando o volume de produção em curso [11].

\section{Just in Time (em Tempo Real)}

Just in Time é a mais reconhecida e utilizada técnica de Lean Production e representa uma filosofia de gestão que procura continuamente eliminar qualquer tipo de desperdício. O Just in Time é uma técnica que permite ter "o material necessário, no lugar certo, na quantidade exata e no tempo pretendido", permitindo aumentar o volume de produção, aumentar o número de encomendas com os mesmos ativos, reduzir os desperdícios, atrasos e tempos de espera e, por conseguinte, reduzir os custos de produção, melhorar a qualidade dos produtos fabricados, libertar os ativos, aumentar as margens de lucro, reduzir os preços de comercialização e aumentar a produtividade [11].

A metodologia Lean Manufacturing integra nos seus processos a filosofia Manutenção Magra (Lean Maintenance) que, obedecendo aos mesmos princípios, tem como objetivo a optimização da eficiência global, a promoção da melhoria contínua, o aumento da confiabilidade e da disponibilidade dos equipamentos e atingir a meta "zero falhas", o que conduz a um aumento da produtividade, da competitividade e da qualidade dos produtos e à redução significativa dos custos diretos e indiretos, associados à Função Produção e à Função Manutenção.

\section{Indicadores de desempenho da Manutenção}

Segundo [13], os indicadores de manutenção mostram o desempenho do departamento. Permitem mensurar a eficácia dos resultados obtidos por meio das ações tomadas, da mesma maneira que medir os problemas encontrados entre o executado e o programado.

De acordo com [13], "os indicadores de manutenção podem ser descritos como "Dados estatísticos relativos à situação da manutenção, sua performance e o crescimento de sua qualidade e desempenho de suas funções"”.

Para [14], os itens de controle são determinados para simplificar e serem essenciais para definir as ações primordiais. Para garantir tempo totalmente designado para coleta de dados, mantem-se somente um número de indicador, sendo útil e necessário. Promovem melhoria na disponibilidade nos equipamentos e na manutenção, aumentando a produção, expressando resultados como: custo de manutenção e número de falhas por um período.

\section{Performance}

Para que a equipe do departamento de manutenção esteja $100 \%$ alocada, devem-se monitorar minuciosamente as atividades dos técnicos [10]. A performance pode ser definida por meio da equação:

$$
\text { Performance }=\frac{\text { Tempo de ciclo de máquina } \mathrm{x} \text { Total de peças produzidas no mês }}{\text { Tempo Operacional }}
$$

Sendo, conforme [15]:

Tempo de Ciclo de Máquina: tempo em que a máquina está operando na velocidade padrão de um determinado produto;

Total de Peças Produzidas no Mês: número de peças produzidas no período; Paradas.

Tempo Operacional $=$ Tempo Disponível - Tempo de

De acordo com [10], "o desempenho mede a influência das perdas por ociosidade ou pequenas interrupções e "velocidade" de trabalho reduzido (máquina operando abaixo da capacidade)".

Segundo [16], "conforme estudos mundiais em empresas que seguem padrões World Class (Classe Mundial), um nível padrão mundial do indicador de performance é em torno de $95 \%$ ".

\section{Qualidade}

$\mathrm{O}$ indicador mede as perdas de produção, uma vez que os materiais defeituosos não podem ser entregues para os clientes [17].

Para o cálculo da qualidade, é definida por [10], como sendo a equação:

$$
\text { Qualidade }=\frac{\text { (Total de peças }- \text { Total de peças produzidas no mês) }}{\text { Total de Peças }}
$$

Sendo, segundo [15]:

Total de Peças: número de peças produzidas;

Total de Peças com Defeito no Mês: número de peças produzidas que não atenderam as especificações.

De acordo com [10], "A qualidade mede a influência das perdas por peças fora de especificações e quedas de rendimento".

De acordo com [16], "se compararmos com estudos mundiais em empresas que seguem padrões World Class (Classe Mundial), o indicador padrão mundial de qualidade é em torno de 99\%".

\section{OEE - Overall Equipment Effectiveness (Eficiência Global do Equipamento)}

O OEE mede a produtividade da empresa por meio de indicadores e verifica se o maquinário está operando perfeitamente. $\mathrm{O}$ indicador quando ilustrar queda significa que deverá realizar algum tipo de intervenção para que o equipamento volte ao seu funcionamento original [18].

Um índice de OEE de $100 \%$ significa que você fábrica apenas bons produtos, o mais rápido possível, sem paradas 
corretivas. Na linguagem de OEE, isso significa $100 \%$ de disponibilidade, performance e qualidade [19].

De acordo com [16], o OEE é um indicador percentual e é calculado da seguinte maneira, conforme equação:

OEE $=$ Disponibilidade $X$ PerformanceX Qualidade

\section{MTBF - Mean Time Between Failures (Tempo Médio entre Falhas)}

$\mathrm{O}$ indicador representa o tempo médio entre as falhas e o tempo de funcionamento do equipamento, mediante a necessidade da produção até a próxima pane [10]. equação:

Segundo [2], o MTBF pode ser calculado por meio da

$\mathrm{MTBF}=\frac{\text { Horas Disponiveis do Equimapento para a Operação }}{\text { Número de Intervenções Corretivas no Equipamento no Período }}$

\section{Sendo:}

Horas Disponíveis do Equipamento para a Operação = Tempo Disponível - Tempo de Paradas [15].

Número de Intervenções Corretivas no Equipamento no Período: quantidade de intervenções corretivas registradas em um determinado período [20].

\section{Reparar}

MTTR - Mean Time To Repair (Tempo Médio para

Esse indicador representa o tempo que os responsáveis pelos equipamentos levam para repará-los. Ou seja, o tempo indisponível do maquinário após a ocorrência da falha. Considerando compra e medição de materiais, equipamentos em reparo e solicitação de fabricação de peças [10].

De acordo com [2], a equação do MTTR pode ser descrita por meio da equação:

De acordo com [21], "se o valor do MTBF com o passar do tempo for aumentando, será um sinal positivo para a manutenção, pois indica que o número de intervenções corretivas vem diminuindo (...)".

$$
M T T R=\frac{\text { Horas de Indisponibilidade para a Operação }}{\text { Número de Intervenções Corretivas no Período }}
$$

Sendo:

Horas de Indisponibilidade para a Operação: tempo de paradas [15.

Número de Intervenções Corretivas no Período: quantidade de intervenções corretivas registradas em um determinado período [20]

Segundo [21], “(...) quanto menor o MTTR no passar do tempo, melhor o andamento da manutenção, pois os reparos corretivos demonstram ser cada vez menos impactantes na produção".

\section{Criação do Planejamento e Controle de Manutenção}

Gestão das Ordens de Serviços que devem ser elaboradas por um profissional da empresa, e este é indispensável para a realização do Planejamento e Controle da Manutenção (PCM).
Nesta etapa, devem ser acrescentados relatórios e gráficos, com informações extraídas dos indicadores de controle [10].

\section{RESULTADOS E DISCUSSÃO}

\section{Elaboração do Plano}

O plano deve ser elaborado por meio de ferramentas que auxiliam o gerenciamento de tarefas e programas mantenedores. Este tem como função assegurar a eficiência dos processos, a fim de atingir as metas e objetivos já determinados [22].

Neste artigo, foram abordadas por vários autores, a importancia das ferramentas Lean Manufacturing, assim como os Indicadores de Manutenção. A filosofia dessas ferramentas na prática, contribui para um processo mais enxuto, com maior possibilidade de detecção de erros e maior facilidade para solução de problemas nos setores de produção e qualidade. E no setor de manutenção não é diferente.

A ferramenta $5 \mathrm{~S}$, por exemplo, contribui para a organização de ferramentas, ou seja, para que elas estejam sempre nos lugares mais próximos aos mecânicos de manutenção, para que eles possam atender no menor tempo possível as chamadas de paradas de máquinas.

Outra ferramenta a ser abordada neste artigo é o gráfico de Gantt. É uma das ferramentas gerenciais mais utilizadas quando se trata da elaboração de um plano de ação.

Existem diversas ferramentas gerenciais, que foram e ainda são criadas, que são utilizadas com o objetivo de analisar se está sendo executado o programado. $\mathrm{Na}$ manutenção, há uma ferramenta que tem esses propósitos e que se tornou popular no meio industrial chamada Gráfico de Gantt [2].

\section{Gráfico de Gantt}

O diagrama de Gantt é utilizado no processo de planejamento e controle de produção e é uma das ferramentas mais utilizadas nas empresas, para elaboração de cronogramas internos de planejamento e acompanhamento de fabricação [23].

O Gráfico de Gantt é um diagrama de barras, que foi desenvolvido no início do século XX, pelo norte-americano Henry Gantt. Foi utilizado nos empreendimentos do Exército e da Marinha, o que fez com que se tornasse popular no gerenciamento de tarefas [2].

Segundo [24], esse cronograma tem por objetivo a visualização do início e do término das tarefas, sendo que estas são posicionadas a esquerda e suas respectivas barras à direita, que indicam o tamanho de suas durações.

O gráfico é ilustrado por meio de barras horizontais para demostrar o tempo previsto para cada tarefa e o início das subsequentes. Após listar todas as atividades e seus tempos de durações, podem-se incluir nomes dos responsáveis de cada serviço [20].

A tabela 1 possibilita a visualização dos serviços programados na preventiva, seus respectivos horários de início, término e duração total das atividades, nomes dos executantes, responsáveis pelos bloqueios mecânicos e elétricos e auxiliares para parada programada. 
Tabela 1: Exemplo do gráfico de Gantt.

\begin{tabular}{|c|c|c|c|c|c|c|c|c|c|c|c|c|c|c|c|c|c|c|c|c|c|c|}
\hline TRABALHOS & \begin{tabular}{ll|} 
inICI \\
\end{tabular} & Fim & \begin{tabular}{l|l|} 
DURA \\
CAAO \\
\end{tabular} & QUEM & 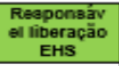 & $\begin{array}{c}\text { STAT } \\
\text { USHO } \\
\text { RA }\end{array}$ & 7 & O7: & $\begin{array}{c}05: \\
30\end{array}$ & $\begin{array}{c}09: \\
30\end{array}$ & \begin{tabular}{l|l}
1 & $10:$ \\
0 & 30 \\
\end{tabular} & \begin{tabular}{|l|l|} 
\\
1 \\
\end{tabular} & $\begin{array}{c}11: \\
30\end{array}$ & & \begin{tabular}{|c|c|}
$12:$ & 1 \\
30 & 3 \\
\end{tabular} & $\begin{array}{c}13: \\
30\end{array}$ & \begin{tabular}{|l|}
1 \\
4 \\
\end{tabular} & \begin{tabular}{l|l|l}
14 & 1 \\
30 & 5 \\
\end{tabular} & \begin{tabular}{|l|l|}
1 & 15 \\
5 & 30 \\
\end{tabular} & \begin{tabular}{|l|}
1 \\
6 \\
\end{tabular} & $\begin{array}{c}16: \\
30 \\
30\end{array}$ & $\frac{1}{7}$ \\
\hline $\begin{array}{l}\text { NAPECGAO NAS } \\
\text { MAGGNESCALES } \\
\text { ANBICID }\end{array}$ & $06: 00$ & 1200 & 04,00 & $\begin{array}{l}\text { LÉOYNOS } \\
\text { ENTTOR }\end{array}$ & & & & & & & & & & & & & & & & & & \\
\hline $\begin{array}{l}\text { REVISÄO E EUMPEZA } \\
\text { NAS LUMNANARIAS DO } \\
\text { STAND } \# 1\end{array}$ & $15: 00$ & $15: 30$ & $00: 30$ & $\begin{array}{l}\text { JOSE } \\
\text { VITOR }\end{array}$ & & & & & & & & & & & & & & & & & & \\
\hline 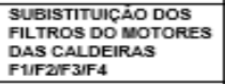 & 14:30 & 16.30 & 02000 & BRUNO & & & & & & & & & & & & & & & & & & \\
\hline $\begin{array}{l}\text { REVISĀO DO SCRAP } \\
\text { CONVEYOR }\end{array}$ & $00: 00$ & $15: 30$ & $07: 30$ & 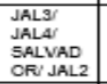 & & $\begin{array}{l}\text { SERV } \\
\text { COA } \\
\text { QUEN } \\
\text { TE }\end{array}$ & & & & & & & & & & & & & & & & \\
\hline 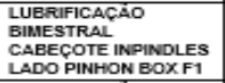 & $08: \infty 0$ & 05.30 & $00: 30$ & ADENIR & & & & & & & & & & & & & & & & & & \\
\hline $\begin{array}{l}\text { LUBREIFICACAंO } \\
\text { BBMESTRAL } \\
\text { CAEECCOTE SPINDLES }\end{array}$ & $06: 30$ & 0900 & $\infty 0: 30$ & ADENIR & & & & & & & & & & & & & & & & & & \\
\hline $\begin{array}{l}\text { PL-OPC-TND-2M-2H-1E } \\
\text { LLO 2H-SACAR-AECK } \\
\text { UP-Tocar coifa da } \\
\text { eacala magnética }\end{array}$ & $\infty 8: 00$ & 1000 & 0200 & $\begin{array}{l}\text { MADSO } \\
\text { N } \\
\text { LEONAR } \\
\text { DO } \\
\text { GABRIEL }\end{array}$ & & & & & & & & & & & & & & & & & & \\
\hline $\begin{array}{l}\text { BLOQUEEIOS E } \\
\text { POSICIONAMAENTOS }\end{array}$ & & & & & & & & & & & & & & & & & & & & & & \\
\hline $\begin{array}{l}\text { BLOQQUUEIOS E } \\
\text { POSICIONAMENTOS } \\
\text { PARA PREVENTIVA }\end{array}$ & $07: 30$ & 06,30 & $01: 00$ & 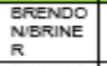 & & & & & & & & & & & & & & & & & & \\
\hline $\begin{array}{l}\text { DESBLOQUEIOS DA } \\
\text { PREVENTTVA }\end{array}$ & $16: 30$ & 1700 & $00: 30$ & $\begin{array}{l}\text { BRENDO } \\
\text { NERINE } \\
\mathbb{R}\end{array}$ & & & & & & & & & & & & & & & & & & \\
\hline $\begin{array}{l}\text { AUIILAR NO } \\
\text { TRABALNOS DA } \\
\text { PREVENTIVA }\end{array}$ & - & 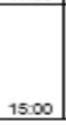 & 08.00 & \begin{tabular}{l|} 
EDUARD \\
ONOAOO \\
BATISTA \\
'OUUGLA \\
SOILA \\
\end{tabular} & & & & & & & & & & & & & & & & & & \\
\hline
\end{tabular}

Fonte: [20].

\section{CONCLUSÃO}

Em uma indústria, a manutenção assume uma função essencial para que os negócios possam enfrentar os desafios que o mercado atual impõe na medida em que a competitividade entre as empresas é cada vez maior, onde a qualidade dos do produto e a flexibilidade do processo produtivo depende diretamente da formação e valorização dos recursos humanos.

Neste sentido, a melhor e mais facilitada capacitação dos recursos humanos em uma empresa vem através de aplicação e treinamento em ferramentas que possam facilitar as atividades diárias, que precisam ser realizadas ao menor tempo possível, atendendo a qualidade esperada pelo cliente. A implementação destes modelos promove a mudança das políticas de manutenção tradicionais, isto é, da manutenção corretiva, que é reativa e funcional, para uma política de manutenção proativa apoiada por ferramentas de otimização e melhoria do desempenho da produtividade, como as abordadas neste artigo.

Visto que a manutenção preventiva auxilia para alcançar os patamares mais importantes para uma empresa, as informações encontradas e explícitas neste artigo mostram que precisam ser disciplinadas quanto à utilização de suas particularidades para alcançar principalmente disponibilidade, performance e qualidade conforme a classe mundial.

\section{REFERÊNCIAS BIBLIOGRÁFICAS}

[1] Cabrita apud Ramos, Pedro. Organização e Gestão da Manutenção Industrial. 2012. Dissertação (Mestrado em Engenharia e Gestão Industrial) - Universidade da Beira Interior, Covilhã-Portugal.

[2] Viana, H. R. G. PCM: Planejamento e Controle da Manutenção. 1. ed. Rio de Janeiro: Qualitymark Editora, 2014.

[3] Neto, T. C. M. A História da Evolução do Sistema de Gestão da Manutenção. Disponível em: $<$ http://www.webartigos.com/artigos/a-historia-da-evolucao-dosistema-de-gestao-de-manutencao/75650/\#ixzz4napRPecr>. Acesso em: 19 jul. 2017.
[4] Alves, I. B. S. et. al. Elaboração e Implementação de um Plano de Manutenção com Auxílio do 5s: Metodologia Aplicada em uma Microempresa. In: Xxix Encontro Nacional De Engenharia De Produção, 10., 2009, Salvador.

[5] Santos, V. A. D. Manual Prático da Manutenção Industrial. 4. ed. São Paulo: Ícone, 2013.

[6] Monteiro, C. I.; Rossi, P. H. L.; Souza, L. R. de. Manutenção Corretiva. Disponível em: <http://wwwp.feb.unesp.br/jcandido/manutencao/Grupo_6.pdf>. Acesso em: 17 jul. 2017.

[7] Auras, A. P.; Moro, N. Introdução à Gestão da Manutenção. Disponível em: <http://norbertocefetsc.pro.br/elm/wpcontent/uploads/2014/12/manutencao.pdf $>$. Acesso em: 24 set. 2016.

[8] Associação Brasileira De Normas Técnicas. NBR 5462: Confiabilidade e Mantenabilidade. Rio de Janeiro, 1994.

[9] Fidelis, N. T. S. et. al. O Papel da Manutenção Autônoma no Processo de Implantação da TPM em uma Empresa do setor Automobilístico. In: XXXV Encontro Nacional De Engenharia De Produção, 10., 2015, Fortaleza.

[10] Pereira, M. G. Engenharia de Manutenção: Teoria e Prática. 2. ed. Rio de Janeiro: Editora Ciência Moderna, 2011.

[11] Ramos, Pedro. Organização e Gestão da Manutenção Industrial. 2012. Dissertação (Mestrado em Engenharia e Gestão Industrial) - Universidade da Beira Interior, Covilhã-Portugal.

[12] Assunção apud Ramos, Pedro. Organização e Gestão da Manutenção Industrial. 2012. Dissertação (Mestrado em Engenharia e Gestão Industrial) - Universidade da Beira Interior, Covilhã-Portugal.

[13] Kardec apud Koch et al. (2013) Koch, S. et al. Análise e Introdução de Técnicas de Manutenção de Classe Mundial no Setor 
de Manutenção Elétrica para Aumentar a Disponibilidade de Equipamentos. In: II WSPI - Workshop Em Sistemas E Processos Industriais, 05., 2013, Santa Cruz do Sul. Conhecimento como aliado às novas tecnologias para Otimização de Processos. Santa Cruz do Sul: Universidade de Santa Cruz do Sul, 2013. p. 1-10.

[14] Koch, S. et al. Análise e Introdução de Técnicas de Manutenção de Classe Mundial no Setor de Manutenção Elétrica para Aumentar a Disponibilidade de Equipamentos. In: II WSPI Workshop Em Sistemas E Processos Industriais, 05., 2013, Santa Cruz do Sul. Conhecimento como aliado às novas tecnologias para Otimização de Processos. Santa Cruz do Sul: Universidade de Santa Cruz do Sul, 2013. p. 1-10.

[15] Vince. O Uso do Tempo: Entendendo o uso do tempo por um equipamento. Disponível em: <http://www.oee.com.br/uso-dotempo/>. Acesso em: 14 dez. 2017.

[16] Silveira, C. B. OEE, Cálculo de Eficiência da Planta e Integração de Sistemas. Disponível em: < https://www.citisystems.com.br/oee-calculo-eficiencia-

equipamentos-integracao-sistemas/>. Acesso em: 25 out. 2017.

[17] Salomâo, A. OEE - Eficiência Global dos Equipamentos. Disponível em: $<$ http://blog.qualidadesimples.com.br/2016/07/05/oee-eficienciaglobal-dos-equipamentos/>. Acesso em: 25 out. 2017.

[18] Cardoso, C. O que é o índice OEE e para que serve? Disponível em: <https://www.automacaoindustrial.info/o-que-e-oindice-oee-e-para-que-serve/>. Acesso em: 25 out. 2017.

[19] Vorne. What is Overall Equipment Effectiveness? Disponível em: <https://www.oee.com/>. Acesso em: 25 out. 2017.

[21] Romanelli, R. Manutenção X Equipamento Novo: como justificar a compra através da avaliação dos indicadores. Disponível em:

https://pt.linkedin.com/pulse/manuten\%C3\%A7\%C3\%A3o-Xequipamento-novo-como-justificar-compra>. Acesso em: $14 \mathrm{dez}$. 2017.

[22] Costa, M. de A. Gestão Estratégica da Manutenção: Uma Oportunidade para Melhorar o Resultado Operacional. 2013. 104f. Monografia (Graduação em Engenharia de Produção) Universidade Federal de Juiz de Fora (UFJF), Juiz de Fora, Minas Gerais, 2013.

[23] Kovaleski, J. L.; Kremer, C. D. Planejamento e Controle dos Processos de Fabricação Metalúrgicos Auxiliado pelo Gráfico de Gantt: Um Estudo de Caso. In: XXXV Encontro Nacional De Engenharia De Produção, 10., 2006, Fortaleza. Fortaleza: Enegep, 2006, p. 1-8.

[24] Stramosk, L. Implementação e Análise de um Processo de Planejamento e Controle da Produção na Construção Civil. 2017. 75f. Monografia (Graduação em Engenharia Civil) - Centro Tecnológico - Departamento de Engenharia Civil - Universidade Federal de Santa Catarina (UFSC), Florianópolis, Santa Catarina, 2017. 